\title{
IT-based planning and control of seaport container terminals and freight transportation systems
}

\author{
Ek Peng Chew • Hans-Otto Günther • \\ Kap Hwan Kim • Herbert Kopfer
}

Published online: 27 May 2010

(C) Springer-Verlag 2010

\section{Introduction}

Over the recent years, maritime container transport has seen tremendous growth rates. As a consequence, the number and capacity of seaport container terminals increased considerably. In today's global freight networks, container terminals not only provide an interface to landside transportation systems but also serve as a hub for the exchange of containers between different maritime shipping lines. Since container terminals represent extremely complex systems with highly dynamic interactions between the various handling, transportation and storage units, and with incomplete knowledge about future events, efficient IT-support and improved logistics control software systems are needed in order to meet the desired performance measures.

\footnotetext{
E. P. Chew

Department of Industrial and Systems Engineering, National University of Singapore, 1 Engineering Drive 2, Singapore 117576, Singapore e-mail: isecep@nus.edu.sg
}

H.-O. Günther $(\bowtie)$

Department of Production Management, Technical University Berlin, Straße des 17. Juni 135, 10623 Berlin, Germany

e-mail: hans-otto.guenther@tu-berlin.de

\section{K. H. Kim}

Department of Industrial Engineering, Pusan National University, Jangjeon-dong, Kumjeong-ku, Busan 609-735, Korea

e-mail:kapkim@pusan.ac.kr

H. Kopfer

Department of Logistics, University of Bremen, Wilhelm-Herbst Straße 5, 28359 Bremen, Germany

e-mail:kopfer@uni-bremen.de 
The primary objective of this special issue is to reflect the recent developments in maritime and landside container transport and to examine research issues concerned with quantitative analysis and decision support for container terminal logistics and freight transportation systems. Eighteen papers have been selected for publication after a thorough peer-review according to the standards of OR Spectrum. These papers fall into four groups which address strategic and operational issues of container terminals, port productivity and inter-port container repositioning, and the interface to on-land transportation systems.

\section{Strategic issues of container terminals}

The first paper by Jörg Wiese, Leena Suhl and Natalia Kliewer presents a mathematical programming based framework for optimizing the layout of container yards. The authors propose a linear model formulation for a rectangular-shaped storage yard and develop a variable neighbourhood search method for dealing with non-rectangular yard configurations. Experimental results show the computational efficiency of the proposed solution methodology.

A simulation study to compare different shapes of berths, namely traditional onesided and indented berths, is presented in the paper by Iris F. A. Vis and Roel G. van Anholt. The simulation model is applied to a realistic scenario taken from a port in the Netherlands. The numerical results provide support for the choice of the type of berth in face of an ongoing increase in the size of container ships.

In their paper, Niels Lang and Albert Veenstra analyze the random arrival of vessels and service processes at container terminals. In particular, they simulate central and decentralized arrival planning strategies under different fuel price scenarios. Their analysis indicates that, for specific scenarios, a central arrival planning strategy provides benefits compared to a decentralized strategy which is common in practice.

Berth arrival planning is also the topic of the paper by Maarten Hendricks, Marco Laumanns, Erjen Lefeber and Jan Tijmen Udding. They develop a practical approach for constructing robust arrival and departure schedules of vessels which minimizes the peak load on quay cranes in the terminal taking arrival window agreements with shipping liners into account. In this way a substantial reduction in total crane capacity can be reached.

Assuming a typical medium-sized container terminal configuration, Liang Ping $K u$, Loo Hay Lee, Ek Peng Chew and Kok Choon Tan investigate the problem of how to find the best yard planning strategy. They propose a generic problem specification with parameterised scenarios and yard planning strategies and formulate a generic mathematical model for determining the optimum weekly yard planning template.

\section{Operation planning and scheduling in container terminals}

The first paper in this group by Pasquale Legato, Rina Mary Mazza and Roberto Trunfio proposes a combined simulation-optimization framework for assigning and scheduling jobs at the available quay cranes in a terminal. To cope with the uncertain and dynamic environment the proposed simulated annealing algorithm is embedded 
into a simulation model which allows a realistic performance estimation of the overall container handling processes.

In the subsequent paper Frank Meisel and Matthias Wichmann develop a novel scheduling methodology for applying double cycling of quay crane loading/unloading operations combined with reshuffling of containers inside the vessel. Numerical experimentation proves that consideration of internal reshuffles leads to a reduction of total vessel handling times compared to a pure double cycling strategy.

A two-phase heuristic and a more elaborative local-search based procedure for realtime scheduling of twin rail-mounted gantry cranes in automated container terminals are proposed by Taejin Park, Ri Choe, Seung Min Ok and Kwang Ryel Ryu. In contrast to existing work, they explicitly consider the rehandling of containers in a block so that the workload of the two stacking cranes at a block can be better balanced and interference more easily avoided.

Automated triple cross-over stacking cranes represent a novel technology for handling containers in a storage block. This type of crane is considered by Ulrich Dorndorf and Frank Schneider who develop an online scheduling strategy which constructs a new schedule for a certain planning horizon whenever a new job arrives or a job is completed. Simulation studies show that the developed scheduling strategy significantly outperforms common rule-based heuristics.

An issue of considerable importance in container terminals is the assignment of transportation jobs to yard trucks in real-time. Matthew E. H. Petering develops a comprehensive discrete event-based simulation model in order to evaluate different real-time control systems for dispatching dual-load yard trucks in a vessel-to-vessel transhipment terminal and compares them in terms of the overall terminal productivity.

In their paper, Loo Hay Lee, Ek Peng Chew, Kok Choon Tan and Yuan Wang develop specific algorithms for dispatching vehicles in a transhipment hub. Specifically, they propose a neighbourhood search procedure and alternatively a genetic algorithm combined with a minimum cost flow network model. Numerical results reveal that the latter method performs superior with respect to the makespan for finishing a given set of jobs at the quay side.

The final paper in this group by Bram Borgman, Eelco van Asperen and Rommert Dekker addresses online rules for container stacking. In a comprehensive simulation study two stacking concepts are compared. The first uses knowledge about container departure times while the second concept focuses on the trade-off between closeness to the exit point of the container and the number of reshuffles. Results show that both concepts provide advantages over benchmark strategies.

\section{Port productivity and inter-port container repositioning}

In the paper by Kevin Cullinane and Tengfei Wang panel data approaches of Data Envelopment Analysis (DEA) are applied for medium to long-term productivity analysis of twenty-five leading container ports. Empirical results reveal that considerable waste exists in container port production. The study also provides a basis indentifying sources of inefficiency in a container port and for developing competitive port strategies. 
Due to the imbalance in global trade the reposition of empty containers from surplus countries to deficit regions has become a challenging problem for most shipping liners. This problem is addressed by Eugene Y. C. Wong, Henry Y. K. Lau and K. L. Mak who propose a novel immunity based evolutionary algorithm to assist shipping liners in repositioning large quantities of empty containers in a cost effective manner.

In their paper, Il-Kyeong Moon, Anh-Dung Do Ngoc and Yun-Su Hur deal with a similar problem. As additional options they include the possibility of leasing and purchasing of containers into their study. To solve the overall cost-minimization problem, they develop a mixed-integer linear optimization model and propose genetic algorithms in order to reduce the computational time.

\section{Interface to on-land transportation systems}

The movement of containers by truck between a customer's location and a container terminal represents the closing link of a container's transportation chain. Ruiyou Zhang, Won Young Yun and Herbert Kopfer model this problem of container transportation in a local area with multiple depots and terminals as a multiple travelling salesman problem with time windows and develop an efficient window-based partition method. Numerical results show that the presented method performs superior compared to a benchmark algorithm.

The impact of the lack of collaboration and coordination in an overseas intermodal transportation chain is investigated in the paper by Carolin Puettmann and Hartmut Stadtler. In order to reduce costs along the entire transportation chain the authors elaborate a coordination scheme which allows the service providers involved to keep their private planning domain with no disclosure of critical data. In addition, the effect of stochastic demand due to time lags in maritime transportation is analysed.

The final paper by Jörn Schönberger investigates demand peak management in a supply chain. Two agents are considered, the supply chain coordinator and the subordinate fleet manager being responsible for providing transportation services. A framework is proposed that controls and adjusts the distribution of decision competence between the two planning agents. Simulation experiments demonstrate that the proposed system is able to increase the reliability of the generated transportation processes.

\section{Concluding remarks}

This special issue has greatly benefited from the cooperation among the authors, reviewers, and editors. We would like to express our sincere thanks to the more than 60 reviewers originating from 20 countries for their excellent and timely refereeing. Last, but not least, we thank all the authors for their contributions which made this special issue possible. 